\title{
A fixed-time diffusion analysis method determines that the three cheV genes of Helicobacter pylori differentially affect motility
}

\author{
Andrew C. Lowenthal, ${ }^{1,2}$ Christopher Simon, ${ }^{3}$ Amber S. Fair, ${ }^{1}$ \\ Khalid Mehmood, ${ }^{1}$ Karianne Terry, ${ }^{1,2}$ Stephanie Anastasia ${ }^{2}$ \\ and Karen M. Ottemann ${ }^{1}$ \\ ${ }^{1}$ Department of Microbiology and Environmental Toxicology, University of California Santa Cruz, \\ Santa Cruz, CA 95064, USA \\ ${ }^{2}$ Department of Molecular, Cell and Developmental Biology, University of California Santa Cruz, \\ Santa Cruz, CA 95064, USA \\ ${ }^{3}$ Department of Applied Mathematics and Statistics, University of California Santa Cruz, Santa Cruz, \\ CA 95064, USA
}

Correspondence

Karen M. Ottemann ottemann@ucsc.edu

Received 30 June 2008

Revised 7 November 2008

Accepted 21 November 2008

\begin{abstract}
Helicobacter pylori is a chemotactic bacterium that has three $\mathrm{CheV}$ proteins in its predicted chemotaxis signal transduction system. CheV proteins contain both CheW- and responseregulator-like domains. To determine the function of these proteins, we developed a fixed-time diffusion method that would quantify bacterial direction change without needing to define particular behaviours, to deal with the many behaviours that swimming $H$. pylori exhibit. We then analysed mutants that had each cheV gene deleted individually and found that the behaviour of each mutant differed substantially from wild-type and the other mutants. cheV1 and cheV2 mutants displayed smooth swimming behaviour, consistent with decreased cellular CheY-P, similar to a cheW mutant. In contrast, the cheV3 mutation had the opposite effect and the mutant cells appeared to change direction frequently. Additional analysis showed that the cheV mutants displayed aberrant behaviour as compared to the wild-type in the soft-agar chemotaxis assay. The soft-agar assay phenotype was less extreme compared to that seen in the fixed-time diffusion model, suggesting that the cheV mutants are able to partially compensate for their defects under some conditions. Each cheV mutant furthermore had defects in mouse colonization that ranged from severe to modest, consistent with a role in chemotaxis. These studies thus show that the $H$. pylori CheV proteins each differently affect swimming behaviour.
\end{abstract}

\section{INTRODUCTION}

Many micro-organisms move in a directed fashion in response to their environment. A common mechanism for movement is via rotary motor organelles called flagella. These motors are regulated by the chemotaxis signal transduction system, which transduces environmental cues into a swimming response. The core of this signal transduction system consists of chemoreceptors, a kinase (CheA), a receptor-kinase coupler (CheW) and a phosphorylatable response regulator (CheY) that controls flagellar rotation (Blair, 1995; Szurmant \& Ordal, 2004).

There are additional proteins that modulate the amount of phosphorylated CheY (CheY-P). Accessory proteins

Abbreviation: FBS, fetal bovine serum.

A supplementary movie showing an example of direction changes in the $H$. pylori cheV1 mutant is available with the online version of this paper. responsible for adaptation and other functions abound, such as methylation of the chemoreceptors by CheR. As more and more prokaryotic genomes have been sequenced, it is becoming clear that motile microbes each have the core signal transduction proteins with a somewhat unique set of these modulator proteins, although it is not yet apparent why particular microbes have particular sets (Blair, 1995; Szurmant \& Ordal, 2004). One such microbe is Helicobacter pylori, a bacterium that uses flagellar motility and chemotaxis to fully colonize animal stomachs (Eaton et al., 1992, 1996; Foynes et al., 2000; Ottemann \& Lowenthal, 2002; Terry et al., 2005). Understanding some of the less-usual attributes of this microbe's chemotactic signal transduction system was the goal of this study.

H. pylori contains homologues of many of the core chemotaxis genes found in other organisms, including cheA (actually a hybrid cheA-Y: contains homologous domains to both Escherichia coli cheA and cheY), cheW and 
cheY. The cheA, cheY and cheW genes are necessary for chemotaxis in soft agar, as expected (Beier et al., 1997; Foynes et al., 2000; Pittman et al., 2001; Terry et al., 2005). $H$. pylori lacks genes coding for the CheR and CheB chemotaxis methylation proteins, and has three $c h e V$ genes. We were particularly curious about the role of the $\mathrm{CheV}$ proteins, as previous studies in Bacillus subtilis had suggested that these proteins can function in adaptation (Karatan et al., 2001).

$\mathrm{CheV}$ proteins are hybrids of $\mathrm{CheW}$ and a response regulator domain similar to CheY. The functions of the $H$. pylori $\mathrm{CheV}$ proteins are not well understood. The first study of these proteins created null mutants of each and analysed them in a chemotaxis soft-agar assay (Pittman et al., 2001). In this assay, bacteria navigate through agar channels to form expanded colonies. Formation of the expanded colonies requires growth, chemotaxis and motility. In this assay, Pittman et al. (2001) found that loss of cheV1 (HP0019) resulted in a strong defect, whereas neither null mutations in cheV2 (HP0616) or cheV3 (HP0393), nor a double mutant of both cheV2 and cheV3, had a detectable phenotype in the same assay. Both cheV2 and cheV3, however, did interfere with chemotaxis when expressed in E. coli, suggesting that the proteins encoded by these genes can interact in the chemotaxis pathway (Pittman et al., 2001). CheV2 has been shown to be phosphorylated in vitro by acetyl phosphate (Pittman et al., 2001), and the isolated CheY domain of all three $\mathrm{CheV}$ proteins can accelerate the dephosphorylation of CheA in vitro, supporting the notion that all CheVs can interact with the CheA chemotaxis protein (Jimenez-Pearson et al., 2005). These studies thus show that CheV1 plays an important but ill-defined role in H. pylori chemotaxis, and that the roles of $\mathrm{CheV} 2$ and $\mathrm{CheV} 3$ in chemotaxis are unclear.

Many other microbes have CheVs, but the best studied is that of B. subtilis. B. subtilis has one cheV and one cheW in its genome. Deletion mutants of either cheW or cheV display reduced ability relative to wild-type to migrate up a concentration gradient of attractant (Rosario et al., 1994). Both cheW and cheV null mutations have an altered basal flagellar bias skewed toward counter-clockwise rotation and thus the smooth swimming/high CheY-P state, although both mutants can still switch flagellar rotation (Karatan et al., 2001; Rosario et al., 1994). A double deletion of $c h e V$ and cheW, on the other hand, resulted in bacteria phenotypically null for chemotaxis, and with a strong bias towards clockwise rotation (low CheY-P) (Rosario et al., 1994). A cheV truncation that retained only the cheW domain could partially complement either a cheW deletion or a cheW cheV double deletion to restore the response to attractant. However this mutant was not able to adapt (Karatan et al., 2001). Likewise, bacteria with a point mutation in $c h e V$, leading to a protein unable to accept phosphates on the CheY domain, also demonstrated reduced ability to adapt (Karatan et al., 2001). Taken together, these studies show two things about CheV's function in B. subtilis. The first is that $\mathrm{CheV}$, along with $\mathrm{CheW}$, is required for full chemoreceptor-CheA coupling. The second is that CheV's CheY domain is needed for adaptation, most likely through deactivating the coupling function of the CheW domain upon acceptance of phosphate (Karatan et al., 2001; Rosario et al., 1994). It is of note that B. subtilis does have the CheR/B proteins, and it has been suggested that $\mathrm{CheV}$ forms an alternative adaptation mechanism (Rao et al., 2008).

Many other bacteria, including Salmonella typhimurium serovar Enterica, have at least one $\mathrm{CheV}$. The cheV of $S$. typhimurium serovar Enterica is part of the flagellar regulon (Frye et al., 2006; McClelland et al., 2001; Wang et al., 2006). cheV deletions in a $\triangle$ cheBR background cause a chemotaxis defect (Wang et al., 2006), while cheV deletions in the wild-type background have no phenotype (Frye et al., 2006).

One challenge for determining the function of the $\mathrm{CheV}$ proteins is the lack of assays for chemotaxis in H. pylori. Historically, much has been learned from observing bacterial swimming behaviour (Berg \& Brown, 1972). We found this approach challenging as $H$. pylori exhibits many types of swimming behaviours, including stops, curves, reversals and speed changes, and so it is hard to determine whether any particular behaviour is most important, and also how to quantify these various behaviours. To overcome this challenge, we developed a method to describe bacterial swimming behaviour mathematically, employing a simple diffusion model. Our model does not require one to define different behaviours. Using this approach, along with others, we were able to determine that each of the $\mathrm{CheV}$ proteins plays a unique role in affecting flagellar rotation.

\section{METHODS}

Bacterial strains. The motile human isolate H. pylori SS1 was used for all experiments. E. coli strain DH10B was used for cloning, and derivatives of E. coli strain RP437 were used for motility studies. All strains are listed in Table 1.

Growth media and chemicals. For solid-medium culture, $H$. pylori was grown on Columbia blood agar (Becton Dickinson) plates with $5 \%$ defibrinated horse blood (Hemostat Laboratories), $5 \mu \mathrm{g}$ trimethoprim $\mathrm{ml}^{-1}, 8 \mu \mathrm{g}$ amphotericin $\mathrm{B} \mathrm{ml}^{-1}, 10 \mu \mathrm{g}$ vancomycin $\mathrm{ml}^{-1}, 50 \mu \mathrm{g}$ cycloheximide $\mathrm{ml}^{-1}, 5 \mu \mathrm{g}$ cefsulodin $\mathrm{ml}^{-1}, 2.5 \mathrm{U}$ polymyxin $\mathrm{B} \mathrm{ml}^{-1}$ and $0.2 \%(\mathrm{w} / \mathrm{v}) \beta$-cyclodextrin (Sigma) (CHBA) at $37{ }^{\circ} \mathrm{C}$ under conditions of $5-10 \% \mathrm{O}_{2}, 10 \% \mathrm{CO}_{2}$ and $80-85 \% \mathrm{~N}_{2}$. All antibiotics were from Sigma or ISC Bioexpress. For liquid culture, H. pylori strains were grown in Brucella broth (Becton Dickinson) with $10 \%$ heat-inactivated fetal bovine serum (FBS) (Gibco) (BB10) on a shaking incubator at $37{ }^{\circ} \mathrm{C}$ under conditions of $5-10 \% \mathrm{O}_{2}, 10 \%$ $\mathrm{CO}_{2}$ and $80-85 \% \mathrm{~N}_{2}$. For selection of mutants, chloramphenicol was used at 5-10 $\mathrm{g} \mathrm{ml}^{-1}$ (H. pylori) or $20 \mu \mathrm{g} \mathrm{ml}^{-1}$ (E. coli).

For long-term storage, a thick 3-5-day growth of $H$. pylori from a CHBA plate was scraped into BB10/1\% (w/v) $\beta$-cyclodextrin $/ 25 \%$ glycerol $/ 5 \%$ DMSO. The cells were dispersed by pipetting and vortexing, then frozen at $-70{ }^{\circ} \mathrm{C}$. 
Table 1. Strains and plasmids used in this study

\begin{tabular}{|c|c|c|c|}
\hline Strain/plasmid & KO no. & Genotype/description & Reference/source \\
\hline \multicolumn{4}{|l|}{ H. pylori } \\
\hline SS1 & & Wild-type & Lee et al. (1997)/J. O'Rourke \\
\hline SS1 cheV1 & KO864 & $\mathrm{SS} 1 \Delta c h e V 1:: c a t_{\mathrm{mut}}$ & This study \\
\hline $\mathrm{SS} 1$ cheV2 & KO865 & $\mathrm{SS} 1 \Delta c h e V 2:: c a t_{\mathrm{mut}}$ & This study \\
\hline SS1 cheV3 & KO856 & $\mathrm{SS} 1 \Delta c h e V 3:: c a t_{\mathrm{mut}}$ & This study \\
\hline SS1 cheY & KO631 & SS1 $\Delta$ cheY:: cat ${ }_{\text {mut }} 102$ & Terry et al. (2005) \\
\hline SS1 cheW & KO852 & SS1 $\Delta$ cheW::aphA3 & Terry et al. (2005) \\
\hline \multicolumn{4}{|l|}{ E. coli } \\
\hline DH10B & & Wild-type & Gibco-BRL \\
\hline RP437 & & Wild-type & D. E. Koshland, Jr \\
\hline RP4315 & & cheY201 & Clegg \& Koshland (1984)/D. E. Koshland, Jr \\
\hline RP3228 & & cheW113 & Parkinson (1978)/D. E. Koshland, Jr \\
\hline \multicolumn{4}{|l|}{ Plasmids } \\
\hline pBluescript (pBS) & & & Stratagene \\
\hline pCat-mut & & $\begin{array}{l}\text { pBS with Campylobacter coli cat gene } \\
\text { lacking terminator }\end{array}$ & Terry et al. (2005) \\
\hline pBS-CheV1 & & pBS : : cheV1 $1_{\text {SS1 }}$ plus 1863 bp flanking seq. & This study \\
\hline pBS-CheV2 & & pBS : : cheV2 $2_{\text {SS1 }}$ plus 1897 bp flanking seq. & This study \\
\hline pBS-CheV3 & & pBS : : cheV $3_{\text {SS1 }}$ plus 1846 bp flanking seq. & This study \\
\hline $\mathrm{pV} 1-c a t_{\mathrm{mut}}$ & & pBS- $\Delta$ cheV1::cat $t_{\mathrm{mut}}$ & This study \\
\hline $\mathrm{pV} 2-c a t_{\mathrm{mut}}$ & & pBS- $\Delta$ cheV2: : cat $t_{\mathrm{mut}}$ & This study \\
\hline $\mathrm{pV} 3-c a t_{\mathrm{mut}}$ & & pBS- $\Delta$ cheV3: : cat $t_{\mathrm{mut}}$ & This study \\
\hline
\end{tabular}

Plasmid preparation was done using kits from Qiagen. For preparation of genomic DNA, DNeasy kits (Qiagen) or Wizard Genomic kits (Promega) were used. All restriction and DNAmodification enzymes were from New England Biolabs or Gibco. Amplification of DNA was carried out using $P f u$ or $P f u$-Turbo polymerases (Stratagene) or Taq polymerase (a generous gift of D. Kellogg). All DNA sequencing was performed by the U. C. Berkeley sequencing facility.

Cloning cheV genes. To isolate the $c h e V$ genes for later use in allelic replacement vectors, each cheV gene and approximately $300 \mathrm{bp}$ of flanking sequences was amplified using PCR and cloned into pBluescript (pBS). There are three cheV genes in each H. pylori genome; they were originally numbered in the J99 genome as cheV1 (hp0019/jhp0017), cheV2 (hp0616/jhp0559) and cheV3 (hp0393/ jhp0988) (Alm et al., 1999), while the 26695 genome does not assign them numbers (Tomb et al., 1997). Primers were designed to clone each cheV using the 26695 genome, and are listed in Table 2, as e.g. cheVlup1 and cheV1down1. Each PCR product was gel purified and treated with T4 kinase (New England Biolabs). These products were then cloned into EcoRV-cut pBluescript, creating the vectors pBSCheV1, pBS-CheV2 and pBS-CheV3. All insertions were verified by restriction analysis and sequencing.

Creation of $\Delta \boldsymbol{c h e V}: \mathbf{c a t}_{\text {mut }}$ mutants. pBS-CheV1, pBS-CheV2 or pBS-CheV3 were used as templates in inverse PCR, in which primers were chosen such that most of the $c h e V$ gene would be deleted from the product (Table 2; primers with iPCR in the name). Specifically, 879 bp were deleted from cheV1, leaving regions coding for five amino acids at the $5^{\prime}$ end, and 30 amino acids at the $3^{\prime}$ end; 660 bp were deleted from cheV2, leaving regions coding for 10 amino acids at the $5^{\prime}$ end and 71 amino acids at the $3^{\prime}$ end; 601 bp were deleted from cheV3, leaving regions coding for 25 amino acids at the $5^{\prime}$ end and 56 amino acids at the $3^{\prime}$ end. Each of these PCR products was gel purified and ligated with a chloramphenicol acetyltransferase gene lacking a terminator that had been excised from the vector pCat-mut
(Terry et al., 2005) using HincII. These ligations created the vectors pV1-cat $t_{\text {mut }}, \mathrm{pV} 2-$ cat $_{\text {mut }}$ and pV3-cat $t_{\text {mut }}$. These plasmids were then transformed into $H$. pylori strain SS1 using natural transformation, and the veracity of each mutant determined by PCR of each locus (data not shown).

RT-PCR analysis of the expression of genes downstream of each cheV. To verify that our cat $t_{\text {mut }}$ insertions had not affected downstream transcription, we carried out reverse transcription (RT)-

Table 2. Primers used in this study

\begin{tabular}{|ll|}
\hline Name & \multicolumn{1}{c|}{ Sequence $\mathbf{( 5}^{\prime} \mathbf{- 3}^{\prime}$ ) } \\
\hline cheV1up1 & ATTTGGGGTTTGTTGGAGGG \\
cheV1down1 & GATCGCTGGGGTTACTTCGC \\
cheV2up1 & AGAAATGATCACTTCGCCCC \\
cheV2down1 & TTTCAATCGGTGGGGTAGCG \\
cheV3up1 & GATGGCTTGAAGTTGTTTGACCGC \\
cheV3down1 & TTGAGCGCCAAAACCAGCCC \\
cheV1iPCRup1 & CGTAACTTGATCAATGCCCGC \\
cheV1iPCRdn1 & GGCCAGGAGTTTGAAGGCCG \\
cheV2iPCRup1 & CGCTTCGTTGTTTAAGTGCAACG \\
cheV2iPCRdn1 & GCTTTTCCAACAAGTTGGCGTGG \\
cheV3iPCRup1 & GGGCATGGGGATGATTTCCCTCT \\
cheV3iPCRdn1 & GGCTATCATTTCTTATTCAAGCTC \\
HP0020_FORWARD & GGCTAAGCTCGCTTTTGAAG \\
HP0020_REVERSE & CTTAGTGATATGATGCCCCC \\
HP0617_FORWARD & GGAGTGAATTTGTGCTAGTG \\
HP0617_REVERSE & CATCTCTAAAGCAACGAGCG \\
HP0392_FORWARD & GATTACGCTAACATGAGCGC \\
HP0392_REVERSE & CTTACCCACTGGTTGCATCC \\
\hline
\end{tabular}


PCR on each downstream gene. A primer set comprising forward and reverse primers that would create $400-500$ bp products was designed for the HP0020 (downstream of cheV1), HP0617 (downstream of cheV2) and HP0392 (downstream of cheV3) genes using the H. pylori 26695 sequence (Table 2). RNA was extracted from plate-grown $H$. pylori SS1 or each of its cheV mutants using Trizol extraction (Invitrogen) followed by RNAeasy Mini kit (Qiagen) and stored at $-20{ }^{\circ} \mathrm{C}$. The RNA was checked on an agarose gel, and $1 \mu \mathrm{g}$ treated with 1-2 U DNase (Invitrogen), following the manufacturer's instructions. For the RT reaction, we used $66 \mathrm{ng}$ DNase-treated RNA and Superscript RT-II Platinum Taq (Invitrogen), following the manufacturer's instructions. Control reactions were done with Taq alone, with its corresponding buffer. Cycling conditions were: (1) initial elongation step at $50{ }^{\circ} \mathrm{C}$ for $30 \mathrm{~min}$; (2) $94{ }^{\circ} \mathrm{C}$ for $2 \mathrm{~min}$; (3) 34 total cycles of $94{ }^{\circ} \mathrm{C}$ for $1 \mathrm{~min}, 50-56{ }^{\circ} \mathrm{C}$ for $1 \mathrm{~min}$ (depending on the primer set), $72{ }^{\circ} \mathrm{C}$ for $1 \mathrm{~min}$; (4) $72{ }^{\circ} \mathrm{C}$ for $3 \mathrm{~min}$.

Fixed-time diffusion motility data collection. $H$. pylori patches on CHBA were used to inoculate BB10. Cultures were typically grown with shaking for $12-16 \mathrm{~h}$ in $10 \% \mathrm{CO}_{2} / 5 \% \mathrm{O}_{2} / 85 \% \mathrm{~N}_{2}$ to an $\mathrm{OD}_{600}$ of $0.05-0.3$, and diluted in fresh BB10 to 30 cells visible in the field. Wet mounts $(50-70 \mu \mathrm{l})$ from these cultures were placed on sittingdrop vapour diffusion slides (Fisher). A minimum of three separate cultures, inoculated from different patches on different days, were used for all $H$. pylori motility analyses.

For E. coli, relevant strains were inoculated as single colonies into tryptone broth, and grown to exponential phase at $30{ }^{\circ} \mathrm{C}$.

Video was taken using a Nikon Eclipse E600 microscope with a Hammatsu 1394 ORCA-285S camera using a $20 \times$ phase-contrast objective. One minute of video was captured with an approximate frame rate of 13 frames $s^{-1}$, with $10-40$ videos taken for each strain or mutant. This video was then analysed using the SimplePCI MTA software package (Compix) to track the bacteria and generate $x, y$ positions of each cell over time.

Fixed-time diffusion data analysis. Before analysis could begin, the data were first filtered to capture only moving bacteria. Nonmotile bacteria were removed first by eliminating any bacteria that moved less than 20 pixels $s^{-1}$. We empirically found the cutoff to work well for differentiating between motile and non-motile $H$. pylori, as well as working for $E$. coli. Fixed-time diffusion requires that all datasets be of the same length of time. We found $4 \mathrm{~s}$ to be a good tradeoff between acquiring enough tracks to analyse, and obtaining data in a linear range. All tracks shorter than $4 \mathrm{~s}$ were removed, while all tracks longer than $4 \mathrm{~s}$ were reduced to $4 \mathrm{~s}$ in length, by ignoring any part of the data after $4 \mathrm{~s}$ had passed. The usual time step for the data was $0.06-0.07 \mathrm{~s}$, and a $4 \mathrm{~s}$ track had 52 points. The abovedescribed filtering of data output from SimplePCI was done by first exporting the $x, y$ positions of each tracked particle into Microsoft Excel formatted files. Data were then imported into R using the gdata package (the $\mathrm{R}$ Project for Statistical Computing, http://www. r-project.org/) (R Development Core Team, 2005). A custom R script was used for filtering the data. Flat text files were exported from $\mathrm{R}$ containing the filtered data.

Next, the filtered position data $(x(t), y(t))$ collected for every bacterium were shifted from the initial position $(x(0), y(0))$ to the origin $(0,0)$ by subtracting $(x(0), y(0))$ from every $(x(t), y(t))$. A transformation to polar coordinates was then done $(x(t), y(t)) \rightarrow(r(t)$, $\theta(t))$. We next calculated the mean square of the radius $\left\langle R^{2}(t)\right\rangle$ for each point in time. A least-square linear regression was then performed on the set of points $\left(\log (t), \log \left(<R^{2}(t)>\right)\right)$. The slope of that curve gave the value of the diffusion exponent $\alpha$. Confidence bands were produced by taking the standard error at the predicted value of $\log <R^{2}(t)>$. This analysis was done in Matlab.
Soft-agar motility assay. Soft-agar plates were made with Brucella broth, $2.5 \%$ FBS and $0.35 \%$ agar. Plates were incubated at room temperature for 3 days before use. Strains were inoculated from CHBA plates into the soft-agar plates using a pipette tip, and migration was measured by measuring the colony diameter each day.

Direct observation of behaviour in soft agar. Low-percentage agar was prepared by autoclaving Brucella broth and $0.42 \%$ agar, and then allowing this solution to cool to $50{ }^{\circ} \mathrm{C}$. For preparing the samples, $900 \mu \mathrm{l}$ was removed into a microcentrifuge tube and mixed with $100 \mu \mathrm{l}$ FBS for a final agar concentration of $\sim 0.38 \%$. This mixture was allowed to cool for $1 \mathrm{~min}$ at room temperature, at which point $20 \mu \mathrm{l}$ of an overnight $H$. pylori culture was added, prepared as described for fixed-time diffusion motility data collection. Tubes were inverted several times to mix the bacteria with the agar solution and $100 \mu \mathrm{l}$ of this mixture was then immediately placed into the well of a sitting-drop vapour diffusion slide (Fisher), and a coverslip quickly added. As heat tends to paralyse H. pylori movement (unpublished observations), under these conditions restoration of movement coincided with solidification of the agar. All observations were made within 15 min of adding bacteria to the agar. Microscopy and video data were collected as described above.

Mouse infection. For all infections, 6-8-week-old female FVB/N mice (Charles River) were housed in an Association for the Assessment and Accreditation of Laboratory Animal Care-accredited facility in microisolator cages with free access to standard food and water. All animal procedures were approved by the Institutional Animal Care and Use Committee, and carried out as described before (Ottemann \& Lowenthal, 2002). For infection, bacteria were grown in BB10 microaerobically with shaking for $16 \mathrm{~h}$, and $1 \times 10^{7}-5 \times 10^{7}$ bacteria in $1 \mathrm{ml} \mathrm{BB10}$ were introduced into each mouse by oral gavage. For competition experiments, a $c h e V$ deletion mutant and the wild-type SS1 strain were mixed together such that there were equal numbers $\left(1 \times 10^{7}-5 \times 10^{7}\right.$ of each strain in $\left.1 \mathrm{ml}\right)$ based on the $\mathrm{OD}_{600}$. All infection amounts were verified by plating the inoculum. For all mouse experiments, the wild-type SS1 was lab passaged the same number of times as the mutant. After 2 weeks, the mice were euthanized by inhalation of $\mathrm{CO}_{2}$, and the bacteria contained in one half stomach were plated onto CHBA with an additional $10 \mu \mathrm{g}$ nalidixic acid $\mathrm{ml}^{-1}$ and $200 \mu \mathrm{g}$ bacitracin $\mathrm{ml}^{-1}$. For competitions, samples of the stomach homogenate were plated onto CHBA and onto CHBA plus chloramphenicol. The number of wild-type bacteria was determined using the following equation: (c.f.u. on CHBA) - (c.f.u. on CHBA-Cm). Statistical analysis was performed using a Student's $t$ test in Microsoft Excel for single-strain infections, or the Wilcoxon, matched-pair signed rank test for competition infections (http:// www.fon.hum.uva.nl/Service/Statistics/Signed_Rank_Test.html).

Protein sequence analysis. Alignments were generated with CLUSTAL W (Thompson et al., 1994), and Boxshade (htpp:// www.ch.embnet.org) was used to visualize them in a readable format.

\section{RESULTS}

\section{The CheV proteins of $H$. pylori contain all the conserved response-regulator and $\mathrm{CheW}$ elements}

H. pylori possesses three $\mathrm{CheV}$ proteins, each composed of fused $\mathrm{CheW}$ and response-regulator/CheY domains, referred to as W- and RR-domains, respectively. Previous analysis of $H$. pylori behaviour found a motility role for CheV1 (Pittman et al., 2001), but could detect no 
phenotype for loss of cheV2 or cheV3. CheV1 is relatively similar to $\mathrm{CheV} 2$ ( $40 \%$ identical, $66 \%$ similar), while CheV3 is less similar (only $24 \%$ identical and $48-49 \%$ similar to either $\mathrm{CheV} 1$ or $\mathrm{CheV} 2$ ). All of the $\mathrm{CheVs}$ retain the phosphorylation active-site residues, as shown by Pittman et al. (2001), but the conservation of the Wdomains had not been examined. As shown in Fig. 1, the $H$. pylori CheVs retain conserved regions known to be important for CheW functions. For example, all of the $H$. pylori $\mathrm{W}$-domains conserve an arginine residue that modulates CheA kinase activity (Boukhvalova et al., 2002; Liu \& Parkinson, 1991). There is also significant charge and residue conservation within $\mathrm{CheW}$ regions responsible for chemoreceptor binding. CheVs also contain several in-frame insertions in both the $\mathrm{W}$ - and RRdomains (Fig. 1 and data not shown), although the function of these insertions is unknown. These findings thus suggest that the $H$. pylori CheVs likely retain CheWlike ability to interact with both chemoreceptors and CheA, and also that they are capable of being phosphorylated.

\section{Deletion of any cheV alters $\boldsymbol{H}$. pylori behaviour in soft agar}

Our alignments suggested that all of the $H$. pylori CheVs should be able to interact with the chemotaxis machinery. We thus sought to thoroughly analyse the motility phenotypes of $H$. pylori lacking each cheV. To that end,
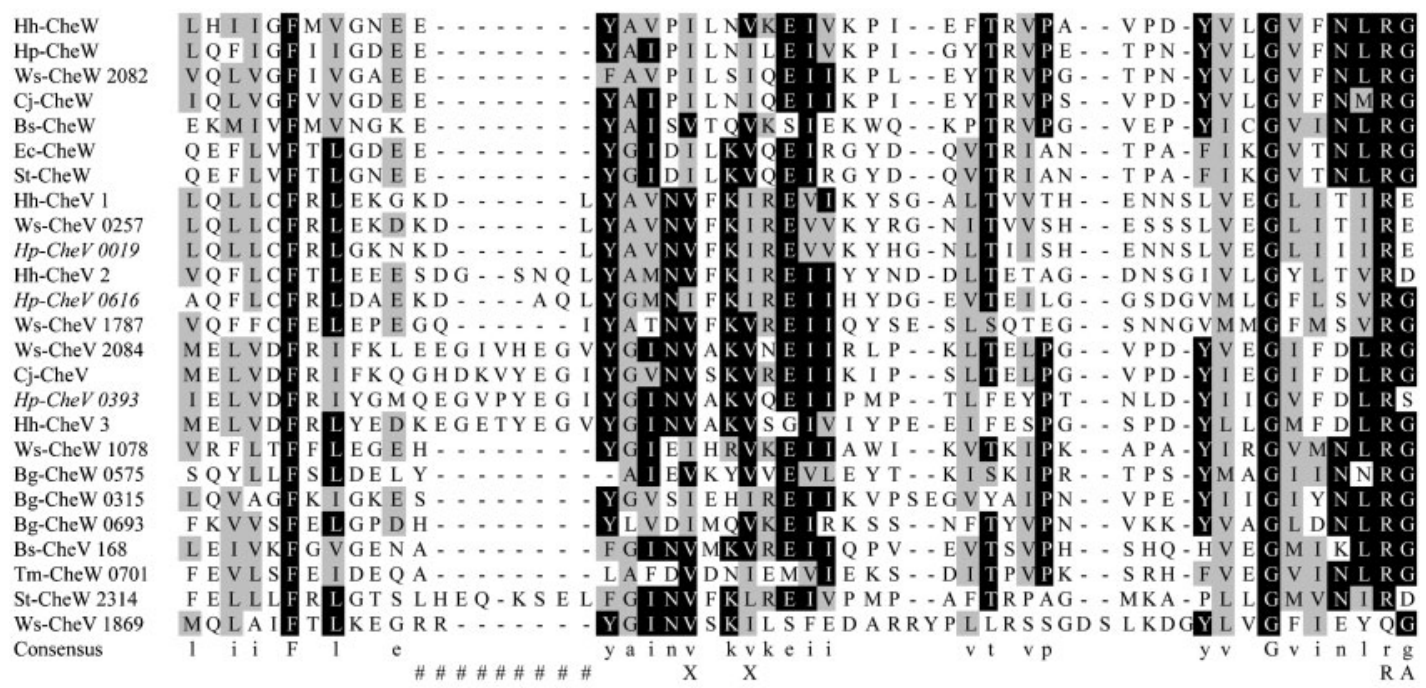

Hh-CheW

Hp-CheW

Ws-CheW 2082

Cj-CheW

Bs-CheW

Ec-CheW

St-CheW

Hh-CheV 1

Ws-CheV 0257

$\mathrm{Hp}$-CheV 0019

Hh-CheV 2

Hp-CheV 0616

Ws-CheV 1787

Ws-CheV 2084

$\mathrm{Cj}-\mathrm{CheV}$

Hp-CheV 0393

Hh-CheV 3

Ws-CheW 1078

Bg-CheW 0575

Bg-CheW 0315

Bg-CheW 0693

Bs-CheV 168

Tm-CheW 0701

St-CheV 2314

Ws-CheV 1869

Consensus

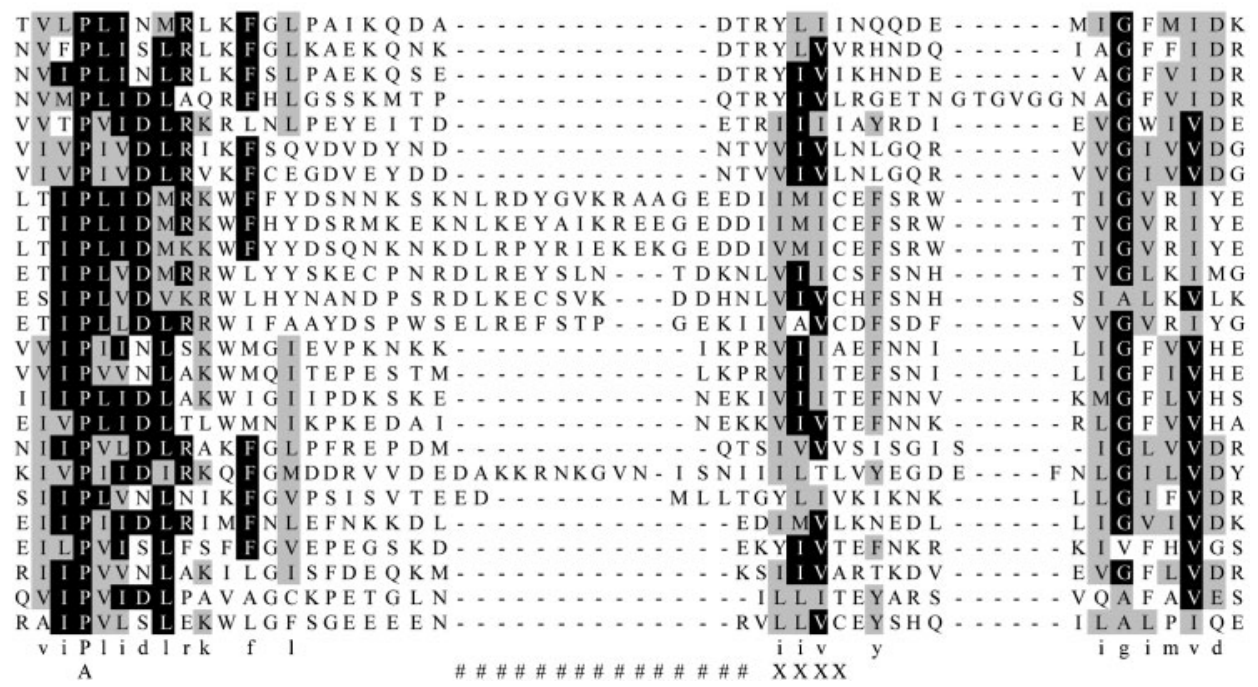

Fig. 1. Alignments of selected CheW-like domains. Labels have the following meanings: Ec is Escherichia coli K-12 MG1655, St is Salmonella enterica serovar Typhimurium LT2, Hp is Helicobacter pylori 26695, Hh is Helicobacter hepaticus ATCC $51449, \mathrm{Cj}$ is Campylobacter jejuni, Tm is Thermotoga maritima MSB8, Ws is Wolinella succinogenes DSMZ 1740, Bs is Bacillus subtilis, $\mathrm{Bg}$ is Borrelia garinii $\mathrm{PBi}$. The symbol \# denotes areas where insertions tend to occur. $\mathrm{X}$ denotes regions involved in chemoreceptor binding. $\mathrm{R}$ is the conserved arginine thought to modulate CheA kinase activity. A are additional conserved residues governing $\mathrm{CheA}-\mathrm{CheW}$ interaction. Black denotes identical residues and grey denotes similar residues. 
we created cheV mutants in the common $H$. pylori strain SS1 by replacing each of the cheV genes individually with a copy of the chloramphenicol acetyltransferase (cat) gene lacking its intrinsic transcriptional terminator. This cat cassette has been shown to completely lack terminating function (Castillo et al., 2008) and to be non-polar in other mutations (Terry et al., 2005). We verified, using RT-PCR, that each downstream gene was expressed as expected (Fig. 2). $c h e V 1$ is predicted to be the first gene in a two-gene operon, cheV2 is predicted to be the first gene in a five-gene operon, and cheV3 is predicted to be the 11th gene in a 13-gene operon. It is of note that cheV3 is followed by the cheA and cheW genes.

We next analysed the behaviour of these mutants, first under non-steady-state conditions in soft-agar assays. For this assay, a small amount of bacteria is inoculated into a rich medium with a low percentage of agar. The gel of the agar creates a three-dimensional structure analogous to a maze, in which bacteria with no ability to change direction cannot navigate. $H$. pylori are believed to migrate out from the starting point in response to changes in nutrient or waste-product status as they carry out metabolism and growth. H. pylori mutants lacking flagellar motility or chemotaxis do not spread significantly through the agar (Eaton et al., 1992; Foynes et al., 2000; Ottemann \&

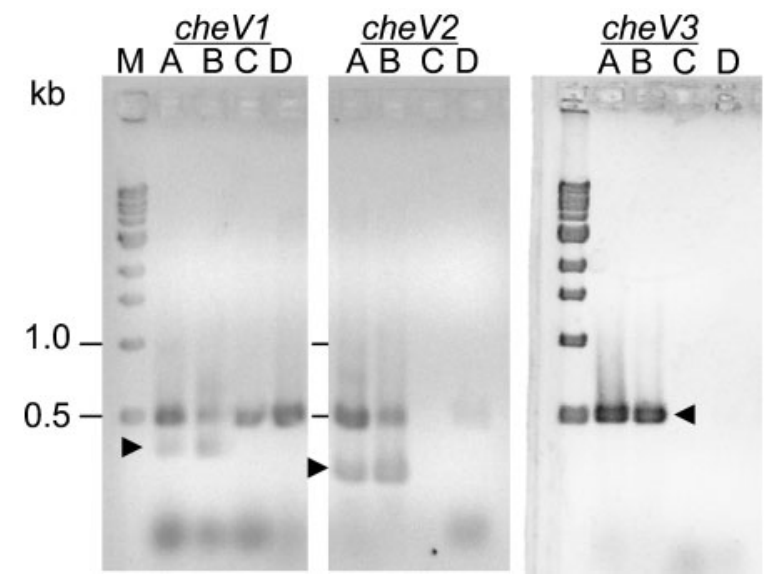

Fig. 2. RT-PCR analysis shows that the genes downstream of each cheV are expressed in cheV mutants. RNA (66 ng per lane) was analysed using RT-PCR and primers designed for the gene downstream of each cheV. Control lanes lack reverse transcriptase but retain Taq. The mutant analysed is indicated above each gel section; arrowheads indicate the RNA-derived PCR product. Each section shows results of RT-PCR of RNA isolated from SS1 wildtype $H$. pylori (A); RT-PCR of RNA isolated from SS1 cheV mutant H. pylori (B); control reaction (Taq only) of RNA isolated from SS1 wild-type $H$. pylori (C); and control reaction (Taq only) of RNA isolated from SS1 cheV mutant $H$. pylori (D). M, size markers. The HP0020 product (downstream of cheV1) is predicted to be $487 \mathrm{bp}$; the HP0617 product (downstream of cheV2) is predicted to be $486 \mathrm{bp}$; the HP0392 product (downstream of cheV3) is predicted to be $450 \mathrm{bp}$.
Lowenthal, 2002; Pittman et al., 2001; Terry et al., 2006). The relative rate of migration, in millimetres per day, reflects the ability of the bacteria to exhibit chemotaxis, swim and grow. The wild-type was found to migrate at a rate of $4.0 \mathrm{~mm}$ per day (Table 3). In agreement with Pittman et al. (2001), we found that deletion of cheV1 resulted in a severe decrease in soft-agar migration (average of $1.5 \mathrm{~mm}$ per day) (Table 3) (Pittman et al., 2001). We observed that both the cheV2 (3.5 mm per day) and cheV3 $(4.3 \mathrm{~mm}$ per day) mutants had subtle but significant alterations in their soft-agar migration, with cheV2 showing a slight decrease in soft-agar motility and cheV3 a slight increase (Table 3). None of these mutants displayed growth defects (data not shown). These results thus suggest that all of the $\mathrm{CheVs}$ might play roles in chemotaxis.

\section{cheV mutants have chemotaxis defects when analysed in a fixed-time diffusion model}

To learn more about the role of each $\mathrm{CheV}$ in $H$. pylori chemotaxis, we developed a mathematical model to describe swimming behaviour based on a fixed-time diffusion measurement. Einstein (1905) first described a mathematical model of small particle diffusion, and we utilized this concept, as well as current theories (Metzler \& Klafter, 2000) to construct our model.

Bacterial motion is characterized by two behaviours: changes in direction and relatively straight swimming. For our analysis, we modelled the motion of swimming bacteria to that of a particle diffusing in medium. From the theory of Brownian motion, we know that after a long enough period of time, the mean square $\left\langle R^{2}(t)\right\rangle$ of the bacterial position is proportional to time, $t$, according to the equation $\left.<R^{2}(t)\right\rangle=D t^{\alpha}$ ( $D$, diffusion constant). We assume that when measurements take place in a sufficiently small amount of time, $T$, the mean square positions of the bacteria will be proportional to $t^{\alpha}$, with $\alpha$ ranging between 1 and 2 . The value of $\alpha$ measures how close the bacterial behaviour is to pure diffusion $(\alpha=1)$. If a bacterium swims straight for a relatively large fraction of the time up to $T$, the value of $\alpha$ will be close to 2. If up to the time $T$ the bacterium swims with frequent direction changes, the value of $\alpha$ will be near 1. Note that the exponent $\alpha$ depends on the value of $T$. If $T$ is too large, any bacteria that change direction will have an $\alpha$ near 1 . Conversely, if $T$ is too small the value of $\alpha$ will be near 2 . We fixed $T$ empirically by observing the trajectories of different bacterial mutants. We chose $T$ of $4 \mathrm{~s}$ because this length of time allowed us both to film sufficient numbers of bacteria (i.e. the bacteria remained within the field and plane of focus), and to generate $\alpha$ values that differed between wild-type and known chemotaxis mutants. Of particular benefit is that the model does not require accurate measurements of tumbling phenomena.

Our basic approach was to capture swimming $H$. pylori using video microscopy and analyse the bacterial beha- 


\section{Table 3. Properties of the $H$. pylori cheV mutants}

Soft-agar migration indicates the colonial expansion rates of $H$. pylori SS1 and mutants on Brucella broth/2.5\% FBS soft agar. The cheW and cheY rates have been reported previously (McGee et al., 2005; Pittman et al., 2001; Terry et al., 2006). The number in parentheses indicates the number of independent colony measurements. Each mutant was compared to a wild-type on the same plate; the migration of $c h e W / c h e Y$ mutants is due to growth only. ND, Not determined. The migration rates of all three $c h e V$ mutants were significantly different from that of wild-type using Student's $t$-test. $\alpha$ is the diffusion exponent in liquid medium. Mouse colonization is the number of H. pylori in the stomach after 2 weeks of infection, with the $95 \%$ confidence interval in parentheses.

\begin{tabular}{|lccc|}
\hline Strain & $\begin{array}{c}\text { Soft-agar migration } \\
\text { (mm per day) }\end{array}$ & $\boldsymbol{\alpha}$ & $\mathbf{1 0}^{-\mathbf{6}} \times$ Mouse colonization \\
\hline H. pylori SS1 (wild-type) & $3.97 \pm 0.01(n=127)$ & $1.6122 \pm 0.0012$ & $3.32(1.53)$ \\
SS1 cheW & $0.5^{\star}$ & $1.8651 \pm 0.0004$ & $0.83^{\star}$ \\
SS1 cheY & $0.5^{\star}$ & $1.9640 \pm 0.0003$ & $1.7^{*}$ \\
SS1 cheV1 & $1.48 \pm 0(97)$ & $1.8986 \pm 0.0011$ & $0.84(0.33)$ \\
SS1 cheV2 & $3.45 \pm 0.01(65)$ & $1.7851 \pm 0.0010$ & $2.52(0.36)$ \\
SS1 cheV3 & $4.31 \pm 0.01(127)$ & $1.1221 \pm 0.0019$ & $2.29(0.74)$ \\
E. coli RP437 & ND & $1.6512 \pm 0.0021$ & - \\
E. coli RP3228 (cheW) & ND & $1.9811 \pm 0.0006$ & - \\
E. coli RP4315 (cheY) & ND & $1.8483 \pm 0.0007$ & - \\
\hline
\end{tabular}

${ }^{\star}$ Data taken from Terry et al. (2005).

viour. Under our conditions, the bacteria and media components freely diffuse, such that the bacteria respond to a relatively constant set of conditions. For these measurements, we took videos of exponential-phase bacteria in their growth medium (BB10) and then used tracking software to generate position $(x, y)$ and time coordinates for each object that was consistent in size with a bacterium. A bacterial 'track' was defined as a set of positional $(x, y)$ and time points that the software assigned to the same bacterium, because a given object was close to the same position in consecutive video frames. The tracking data were filtered to remove non-motile bacteria, and tracks were then trimmed to be $4 \mathrm{~s}$ in length. The positional data were then transformed to move the beginning of each track to the origin $(0,0)$, with all changes in position relative to the origin preserved. The average change in distance over time was used to calculate the diffusion exponent, $\alpha$, for each bacterial strain, as described in Methods and above.

We first tested our quantitative diffusion description with wild-type $E$. coli and found it had a diffusion exponent $\alpha$ of 1.6512 (Table 3). This value is intermediate between 1 and 2 , suggesting that $E$. coli behaviour is set between smooth swimming and changing direction, as expected. We next analysed E. coli with mutations in the chemotaxis genes cheW or cheY. Mutants lacking these proteins constantly swim and almost never change direction (Blair, 1995). Using our fixed-time diffusion method, we found that these mutants were more smooth swimming than wildtype, with $\alpha$ values closer to 2 , as expected (Table 3 ). Thus, our method works and appears sensitive to directional changes of the analysed bacteria.

We next used this methodology to analyse the behaviour of $H$. pylori. Wild-type bacteria again demonstrated an intermediate diffusion exponent $(\alpha=1.6122)$, while the cheY mutant $(\alpha=1.9640)$ and the cheW mutant $(\alpha=1.8651)$ were more smooth swimming (Table 3, Fig. 3). These measurements show that cells bearing the cheY mutant allele were significantly more smooth swimming than those with the cheW mutation. Furthermore, these data support the notion that CheY-P is involved in creating direction changes in $H$. pylori, along the lines of the E. coli model, because mutants unable to make CheY, and hence CheY-P, are smooth swimming. We then extended this analysis to our cheV mutants. Surprisingly, we found that one of them, the cheV3 mutant, was heavily biased towards changing direction $(\alpha=1.1221)$, while the cheV1 mutant $(\alpha=1.8986)$ and the cheV2 mutant $(\alpha=1.7851)$ were both smooth swimming (Table 3, Fig. 3). This analysis suggests that all three CheVs participate in controlling flagellar rotation, but that they play different roles. Loss of either of CheV1 or CheV2 is similar to loss of $\mathrm{CheW}$ or $\mathrm{CheY}$, and results in cells that are less able to change direction than is the wildtype. Loss of $\mathrm{CheV} 3$, on the other hand, causes cells to change direction much more frequently than wild-type, akin to mutants that have elevated CheY-P.

We were somewhat puzzled by the apparently different phenotypes of the cheV mutants in the soft-agar assay versus the fixed-time diffusion swimming assay. For example, the cheV2 mutant rarely changes direction in the diffusion assay, but traverses the soft-agar plates relatively well. Other straight-swimming mutants such as cheW, cheA or cheY cannot migrate through these soft-agar plates (Beier et al., 1997; Foynes et al., 2000; Pittman et al., 2001), suggesting that the cheV mutants might display condition-dependent phenotypes in the soft-agar assay. To assess the actual swimming behaviour of the mutants in the 
WT

$\alpha=1.6122$

cheY

$\alpha=1.9640$

cheW

$\alpha=1.8651$
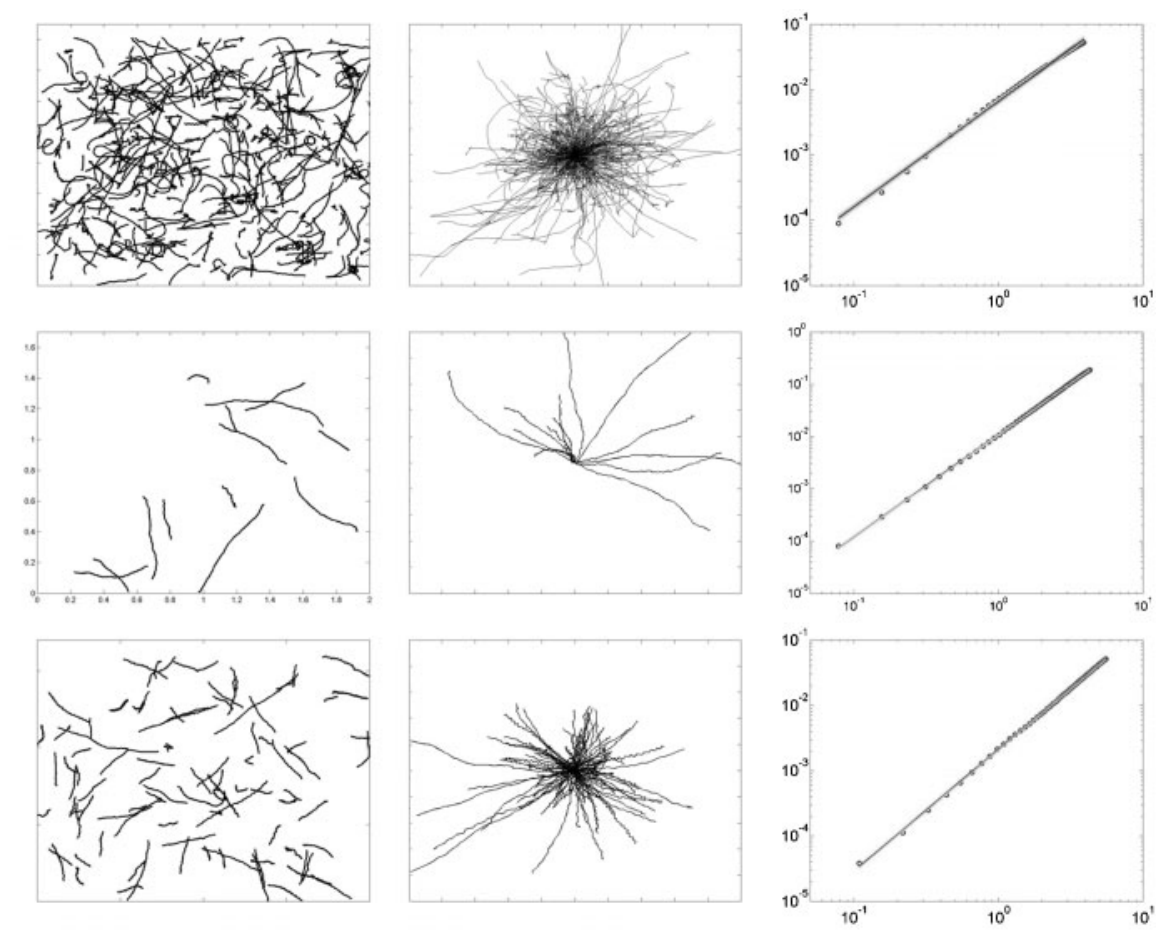

chev1
$\alpha=1.8975$
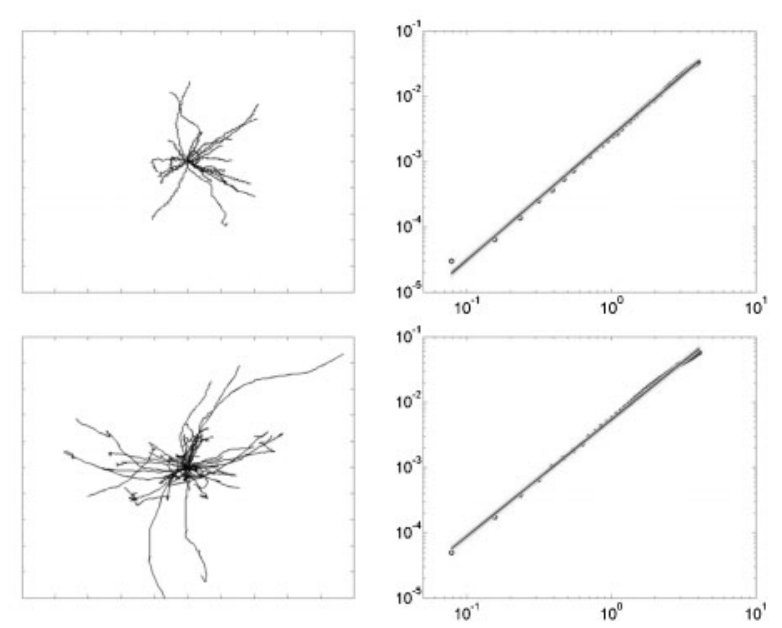

cheV2

$\alpha=1.7851$
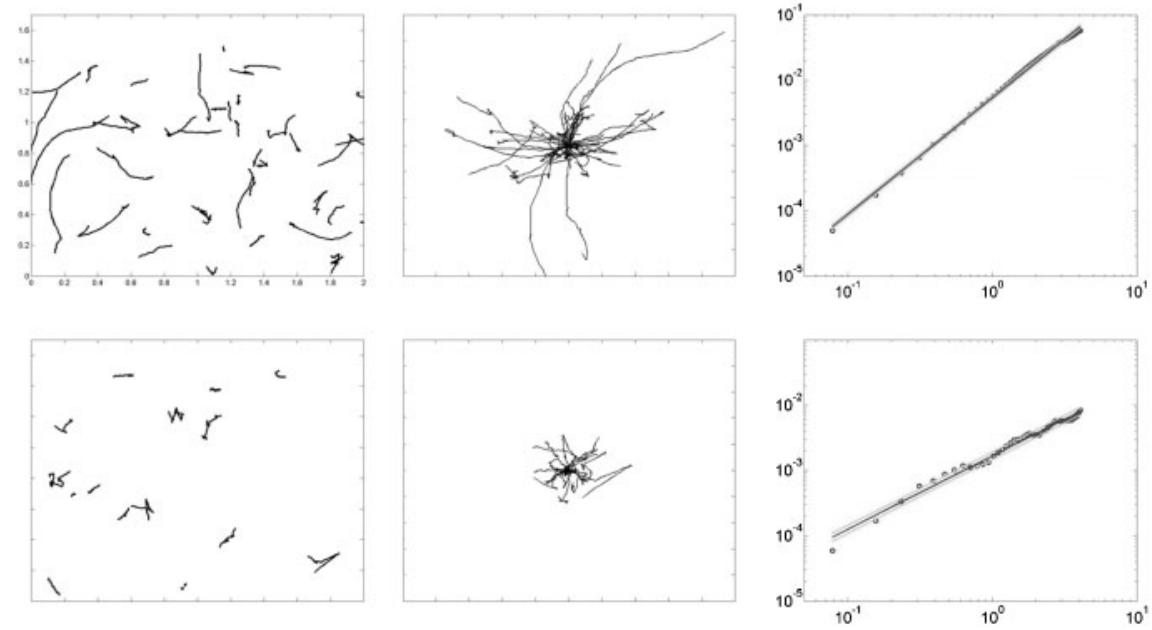

Fig. 3. Motility patterns of $H$. pylori SS 1 and its chemotaxis mutants. The left panels show the un-rotated $4 \mathrm{~s}$ swimming tracks of the strain indicated on the left; each is set to the same scale. The middle panels show the same tracks rotated to originate from the origin; each is set to the same scale. The right panels show graphs of $\left\langle R^{2}(t)>v s\right.$ time (s) for these tracks. The diffusion exponent, $\alpha$, is derived from the slope of the line and is given on the left. Light grey lines on each side of the plotted lines denote error. The number of tracks analysed for each strain is as follows: WT, >50; cheY, 13; cheW, >30; cheV1, 21; cheV2, 18; cheV3, 18. 
soft-agar assay, we created miniature versions of this assay in hanging-drop microscope slides. We were able to observe $H$. pylori in these mini soft-agar assays, and saw that the wild-type and each of the cheV mutants was able to reverse directions. An example with the $H$. pylori cheV1 mutant is shown in the supplementary movie available with the online version of this paper. As $H$. pylori cells are elongated and not spherical, it was easy to observe direction changes, although differences between the individual mutant strains were too subtle to quantify in this particular assay. The cheY mutant was never observed to move in the agar, consistent with it being immobilized at the end of channels (data not shown). However, smooth swimming motility was noted in the agar close to glass before solidification was complete, indicating that the mutant was capable of swimming in this assay.

\section{CheV proteins play roles in mouse infection}

As it was shown previously that chemotaxis is important for $H$. pylori infection of mice, we analysed how the cheV mutants would fare in the stomach. For these experiments we infected mice with $\sim 5 \times 10^{7}$ cells of each cheV mutant. After 2 weeks of infection, we analysed the number of $H$. pylori in the stomach, and compared this amount to mice infected with wild-type alone. Infection with wild-type SS1 bacteria resulted in colonization at an average of $3.32 \times 10^{6}$ c.f.u. per g stomach (Table 3). Both the cheV2 and cheV3 mutants were able to infect to levels that were not different from wild-type, while the cheV1 mutant had a somewhat decreased ability to colonize mouse stomachs (Table 3 ). As stated above, these mutants did not display in vitro growth rate differences.

A more sensitive assay of colonization ability was employed by infecting the mice with mixtures of wild-type and one of the cheV mutants. Again, we allowed the infection to proceed for 2 weeks and determined the number of mutant and wild-type bacteria remaining in the stomach. Using this assay, we found that the cheV1 mutant had the most severe defect, and was outcompeted $>1000$ fold by the wild-type (Fig. 4). The cheV2 mutant was slightly and significantly outcompeted (1.8-fold). Strains lacking cheV3 were also significantly outcompeted ( 8.5 -fold) by wildtype. Thus, all three of the $c h e V$ genes appear to play roles in mouse-stomach colonization.

\section{DISCUSSION}

CheV proteins are widespread thoughout bacteria but remain relatively understudied. $H$. pylori is unusual in that it has three CheVs with only one set of the core chemotaxis proteins, and no CheR or CheB. We have shown here that the H. pylori CheVs all affect flagellar rotation, and play distinct roles in the process.

Wild-type $H$. pylori exhibits many different swimming behaviours which could affect chemotaxis. These beha-

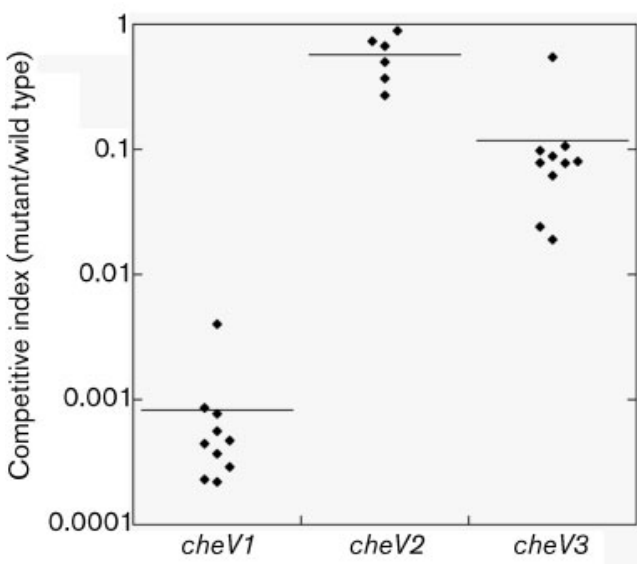

Fig. 4. Competitive index $(\mathrm{Cl})$ of $H$. pylori SS1 cheV mutants coinfected with wild-type (WT). $\mathrm{Cl}=$ mutant/WT output $_{\text {divided by }}$ mutant/WT input. $\mathrm{A} \mathrm{Cl}$ of 1 indicates a mutant with the same infection ability as WT. For cheV1, $n=10$ and the $P$-value is $<0.01$. For cheV2, $n=6$ and the $P$-value is $<0.05$. For cheV3, $n=10$ and the $P$-value is $<0.01$.

viours include reversals, stops with and without reorientations, and changes in velocity. As it was unclear which of the many observed $H$. pylori behaviours was important for direction changes, and the importance of any particular behaviour could vary between wild-type and a mutant, we sought a method in which neither the method of direction change nor the velocity of bacteria would bias the results. Fixed-time diffusion was chosen as it fits these requirements, is intuitive, and is sensitive to small changes in behaviour. Using the equation $\left\langle R^{2}(t)\right\rangle=D t^{\alpha}$, where the coefficient $\alpha$ describes the behaviour as it relates to Brownian ( $\alpha$ close to 1 , or many changes in direction) or ballistic ( $\alpha$ close to 2 , or no changes in direction), we were able to describe the behaviour of motile bacteria without the need to assume the extent to which various behaviours contributed to direction change. Use of this method facilitated our analysis of the roles for the CheV proteins.

Using the fixed-time diffusion approach to study $H$. pylori behaviour in liquid media, in which bacteria and media components diffuse freely, we found that each of the three CheVs plays a role in affecting flagellar rotation. Mutants lacking CheV1 are extremely smooth swimming, with only cheY mutants having a more smooth bias. Mutants lacking CheV2 are very smooth swimming as well. The soft-agar phenotypes mirror these phenotypes, albeit imperfectly. The cheV1 mutants have a significant soft-agar defect, while mutants lacking cheV2 have only a marginal defect. The finding that the cheV2 mutant soft-agar phenotype is not as severe as might be expected from the diffusion exponent suggests that there could be a compensatory occurrence in the soft-agar assay. Similarly, a B. subtilis cheV mutant shows altered steady-state bias and time to adapt but retains the ability to adapt (Garrity \& Ordal, 
1995; Rosario et al., 1994). The two chemotaxis assays used here have quite different conditions. For the diffusion analysis, the assay is relatively short (in the order of minutes), does not require the bacteria to generate a gradient and occurs in a liquid milieu. The soft-agar assay, in contrast, takes days, requires bacterial metabolism and growth, and occurs in a gel-like medium that has barriers to bacterial movement. Bacteria do not form chemical gradients in liquid culture, while the relatively slow forward progress in agar may allow them to generate a gradient through metabolism of attractants or release of repellants. We found that the two smooth-biased cheV deletion mutants were able to reverse direction in soft agar even when they had been embedded in it for a short period of time. One explanation is thus that the smooth-biased cheV1 and cheV2 mutants are capable of changing direction when a large enough chemical gradient is applied.

Mutants lacking cheV3 display a strikingly different phenotype from those of the other cheV mutants. We found that these mutants switched direction frequently and migrated in soft agar at the same rate as wild-type. E. coli mutants with similarly elevated directional changes, such as those lacking cheZ or cheB, do better in the soft-agar assay than their smooth-locked counterparts, although not better than wild-type (Wolfe \& Berg, 1989). Strictly smoothswimming cells would be expected to get stuck in the softagar channels, while tumbly mutants might be able to make their way through the matrix to some degree. It is possible that the swimming bias of this mutant is slightly modulated by conditions in the soft-agar assay, as hypothesized for the cheV2 mutant above. Alternatively, because $H$. pylori migrates about 25 times slower in the soft-agar assay than does E. coli, perhaps tumble-bias mutants fare better in this bacterium.

We further utilized mouse-infection phenotypes to determine how the cheV mutants compare with known chemotaxis mutants. The mouse-infection phenotypes mirrored the soft-agar phenotypes more closely than they mirrored the liquid-swimming phenotypes. We hypothesize that the cheV mutants are able to adjust their swim/ tumble biases under some conditions, such as in soft agar and the mouse stomach. The cheV1 mutant was the most impaired cheV mutant in soft agar and similarly was the most impaired in the mouse stomach. This phenotype is similar to other swim-bias mutants such as those lacking cheA, cheW or cheY (Terry et al., 2005). The cheV2 mutant was only moderately impaired in the soft agar, and had only a slight defect in the mouse model. This phenotype suggests that these mutants are able to change directions under conditions encountered in both settings. Mutants lacking cheV3 are moderately outcompeted in the mouse model. These mutants have an extreme bias towards frequent direction changes and migrate somewhat more quickly than wild-type in soft agar. The behaviour in mice is consistent with only moderately impaired chemotaxis in this setting, although it has not been determined how $H$. pylori mutants with a non-swim bias fare in this setting.
Vibrio cholerae tumble-bias mutants perform much better than swim-bias mutants during infection (Butler \& Camilli, 2004).

In conclusion, we have shown that each $H$. pylori $\mathrm{CheV}$ affects swimming behaviour, as postulated by others (JimenezPearson et al., 2005; Pittman et al., 2001; Szurmant \& Ordal, 2004). We favour a model in which each $\mathrm{CheV}$ performs chemoreceptor-CheA kinase coupling that is regulatable by the phosphorylation state of the CheY domain of the $\mathrm{CheV}$, as in B. subtilis (Rao et al., 2008). H. pylori CheVs, however, can exist in either CheA-activating or CheA-deactivating states, and phosphorylation switches them toward the opposite form. Indeed, two of the $H$. pylori CheVs seem to activate CheA, while the third seems to deactivate CheA. We have noticed that the $\mathrm{CheV}$ proteins fall into families with insertions conserved in size, position and sequence within the Helicobacteraceae (Fig. 1). These sequences may have some relevance to function, as they are found close to the chemoreceptor binding pocket (data not shown).

It seems most likely that all the CheVs plus CheW exist as couplers in a presumed chemoreceptor supercomplex. Based on mutant phenotypes, CheW plays a pivotal role (Pittman et al., 2001; Terry et al., 2005). The phosphorylation state of each $\mathrm{CheV}$ may be a way for $\mathrm{H}$. pylori to adapt, as shown with the B. subtilis $\mathrm{CheV}$ (Karatan et al., 2001; Rao et al., 2008). This role in $H$. pylori seems especially likely given that there seem to be no other proteins involved in adaptation in this microbe.

\section{ACKNOWLEDGEMENTS}

We are grateful to David Blair for suggesting the use of vapour diffusion slides to avoid complications of bacterial swimming behaviour close to glass, Andrea Castillo for technical suggestions, and Chad Saltikov, Fitnat Yildiz and members of the Ottemann Lab for comments on the manuscript and creative discussion. The project described was supported by grant number 050000 (to K. M. O.) from the National Institutes of Allergy and Infectious Disease (NIAID) at the National Institutes of Health. Its contents are solely the responsibility of the authors and do not necessarily represent the official views of the NIH.

\section{REFERENCES}

Alm, R. A., Ling, L. S., Moir, D. T., King, B. L., Brown, E. D., Doig, P. C., Smith, D. R., Noonan, B., Guild, B. C. \& other authors (1999). Genomic-sequence comparison of two unrelated isolates of the human gastric pathogen Helicobacter pylori. Nature 397, 176-180.

Beier, D., Spohn, G., Rappuoli, R. \& Scarlato, V. (1997). Identification and characterization of an operon of Helicobacter pylori that is involved in motility and stress adaptation. J Bacteriol 179, 4676-4683.

Berg, H. C. \& Brown, D. A. (1972). Chemotaxis in Escherichia coli analysed by three-dimensional tracking. Nature 239, 500-504.

Blair, D. F. (1995). How bacteria sense and swim. Annu Rev Microbiol 49, 489-522.

Boukhvalova, M. S., Dahlquist, F. W. \& Stewart, R. C. (2002). CheW binding interactions with CheA and Tar - importance for chemotaxis signaling in Escherichia coli. J Biol Chem 277, 22251-22259. 
Butler, S. M. \& Camilli, A. (2004). Both chemotaxis and net motility greatly influence the infectivity of Vibrio cholerae. Proc Natl Acad Sci U S A 101, 5018-5023.

Castillo, A. R., Arevalo, S. S., Woodruff, A. J. \& Ottemann, K. M. (2008). Experimental analysis of Helicobacter pylori transcriptional terminators suggests this microbe uses both intrinsic and factordependent termination. Mol Microbiol 67, 155-170.

Clegg, D. O. \& Koshland, D. E. (1984). The role of a signaling protein in bacterial sensing: behavioral effects of increased gene expression. Proc Natl Acad Sci U S A 81, 5056-5060.

Eaton, K. A., Morgan, D. R. \& Krakowka, S. (1992). Motility as a factor in the colonisation of gnotobiotic piglets by Helicobacter pylori. J Med Microbiol 37, 123-127.

Eaton, K. A., Suerbaum, S., Josenhans, C. \& Krakowka, S. (1996), Colonization of gnotobiotic piglets by Helicobacter pylori deficient in two flagellin genes. Infect Immun 64, 2445-2448.

Einstein, A. (1905). On the motion of small particles suspended in liquids at rest required by the molecular-kinetic theory of heat. Annalen der Physik 17, 549-560.

Foynes, S., Dorrell, N., Ward, S. J., Stabler, R. A., McColm, A. A., Rycroft, A. N. \& Wren, B. W. (2000). Helicobacter pylori possesses two CheY response regulators and a histidine kinase sensor, CheA, which are essential for chemotaxis and colonization of the gastric mucosa. Infect Immun 68, 2016-2023.

Frye, J., Karlinsey, J. E., Felise, H. R., Marzolf, B., Dowidar, N., McClelland, M. \& Hughes, K. T. (2006). Identification of new flagellar genes of Salmonella enterica serovar Typhimurium. J Bacteriol 188, 2233-2243.

Garrity, L. F. \& Ordal, G. W. (1995). Chemotaxis in Bacillus subtilis: how bacteria monitor environmental signals. Pharmacol Ther 68, 87104.

Jimenez-Pearson, M. A., Delany, I., Scarlato, V. \& Beier, D. (2005). Phosphate flow in the chemotactic response system of Helicobacter pylori. Microbiology 151, 3299-3311.

Karatan, E., Saulmon, M. M., Bunn, M. W. \& Ordal, G. W. (2001). Phosphorylation of the response regulator $\mathrm{CheV}$ is required for adaptation to attractants during Bacillus subtilis chemotaxis. J Biol Chem 276, 43618-43626.

Lee, A., O'Rourke, J., Ungria, M. C. D., Robertson, B., Daskalopoulos, G. \& Dixon, M. F. (1997). A standardized mouse model of Helicobacter pylori infection: introducing the Sydney strain. Gastroenterology 112, 1386-1397.

Liu, J. D. \& Parkinson, J. S. (1991). Genetic evidence for interaction between the CheW and Tsr proteins during chemoreceptor signaling by Escherichia coli. J Bacteriol 173, 4941-4951.

McClelland, M., Sanderson, K. E., Spieth, J., Clifton, S. W., Latreille, P., Courtney, L., Porwollik, S., Ali, J., Dante, M. \& other authors (2001). Complete genome sequence of Salmonella enterica serovar Typhimurium LT2. Nature 413, 852-856.
McGee, D. J., Langford, M. L., Watson, E. L., Carter, J. E., Chen, Y. T. \& Ottemann, K. M. (2005). Colonization and inflammation deficiencies in Mongolian gerbils infected by Helicobacter pylori chemotaxis mutants. Infect Immun 73, 1820-1827.

Metzler, R. \& Klafter, J. (2000). The random walk's guide to anomalous diffusion: a fractional dynamics approach. Phys Rep 399, $1-77$.

Ottemann, K. M. \& Lowenthal, A. C. (2002). Helicobacter pylori uses motility for initial colonization and to attain robust infection. Infect Immun 70, 1984-1990.

Parkinson, J. S. (1978). Complementation analysis and deletion mapping of Escherichia coli mutants defective in chemotaxis. $J$ Bacteriol 135, 45-53.

Pittman, M. S., Goodwin, M. \& Kelly, D. J. (2001). Chemotaxis in the human gastric pathogen Helicobacter pylori: different roles for CheW and the three $\mathrm{CheV}$ paralogues, and evidence for $\mathrm{CheV} 2$ phosphorylation. Microbiology 147, 2493-2504.

Rao, C. V., Glekas, G. D. \& Ordal, G. W. (2008). The three adaptation systems of Bacillus subtilis chemotaxis. Trends Microbiol 16, 480-487.

R Development Core Team (2005). R: a Language and Environment For Statistical Computing. Vienna, Austria: R Foundation for Statistical Computing.

Rosario, M. M., Fredrick, K. L., Ordal, G. W. \& Helmann, J. D. (1994). Chemotaxis in Bacillus subtilis requires either of two functionally redundant CheW homologs. J Bacteriol 176, 2736-2739.

Szurmant, H. \& Ordal, G. W. (2004). Diversity in chemotaxis mechanisms among the bacteria and archaea. Microbiol Mol Biol Rev 68, 301-319.

Terry, K., Williams, S. M., Connolly, L. \& Ottemann, K. M. (2005). Chemotaxis plays multiple roles during Helicobacter pylori animal infection. Infect Immun 73, 803-811.

Terry, K., Go, A. C. \& Ottemann, K. M. (2006). Proteomic mapping of a suppressor of non-chemotactic cheW mutants reveals that Helicobacter pylori contains a new chemotaxis protein. Mol Microbiol 61, 871-882.

Thompson, J. D., Higgins, D. G. \& Gibson, T. J. (1994). CLUSTAL W: improving the sensitivity of progressive multiple sequence alignment through sequence weighting, position-specific gap penalties and weight matrix choice. Nucleic Acids Res 22, 4673-4680.

Tomb, J. F., White, O., Kerlavage, A. R., Clayton, R. A., Sutton, G. G., Fleischmann, R. D., Ketchum, K. A., Klenk, H. P., Gill, S. \& other authors (1997). The complete genome sequence of the gastric pathogen Helicobacter pylori. Nature 388, 539-547.

Wang, Q., Mariconda, S., Suzuki, A., McClelland, M. \& Harshey, R. M. (2006). Uncovering a large set of genes that affect surface motility in Salmonella enterica serovar Typhimurium. J Bacteriol 188, 7981-7984.

Wolfe, A. J. \& Berg, H. C. (1989). Migration of bacteria in semisolid agar. Proc Natl Acad Sci U S A 86, 6973-6977.

Edited by: J. G. Shaw 